\title{
The Hydrogen Atom in Combined Electric and Magnetic Fields with Arbitrary Mutual Orientations
}

\author{
Jörg Main, Michael Schwacke, and Günter Wunner \\ Institut für Theoretische Physik I, Ruhr-Universität Bochum, D-44780 Bochum, Germany
}

(August 28, 1997)

For the hydrogen atom in combined magnetic and electric fields we investigate the dependence of the quantum spectra, classical dynamics, and statistical distributions of energy levels on the mutual orientation of the two external fields. Resonance energies and oscillator strengths are obtained by exact diagonalization of the Hamiltonian in a complete basis set, even far above the ionization threshold. At high excitation energies around the Stark saddle point the eigenenergies exhibit strong level repulsions when the angle between the fields is varied. The large avoided crossings occur between states with the same approximately conserved principal quantum number, $n$, and this intramanifold mixing of states cannot be explained, not even qualitatively, by conventional perturbation theory. However, it is well reproduced by an extended perturbation theory which takes into account all couplings between the angular momentum and Runge-Lenz vector. The large avoided crossings are interpreted as a quantum manifestation of classical intramanifold chaos. This interpretation is supported by both classical Poincaré surfaces of section, which reveal a mixed regular-chaotic intramanifold dynamics, and the statistical analysis of nearest-neighbor-spacing distributions.

PACS numbers: 32.60.+i, 03.65.Sq, 05.45.+b, 32.70.Cs

\section{INTRODUCTION}

The effects of external electric and magnetic fields on atomic spectra is a fundamental question of atomic physics. However, up to now most investigations have concentrated on atoms in only one of the two fields, or on selected mutual orientations such as parallel or perpendicular fields. For example, Rydberg atoms in magnetic fields are nonintegrable systems and have been shown to be ideally suited for studies of the quantum manifestations of classical chaos [1] 3]. Because of the cylindrical symmetry around the magnetic field axis, one component of the angular momentum is conserved, and the problem is nonseparable in two degrees of freedom. The cylindrical symmetry is broken in combined nonparallel magnetic and electric fields, and the system becomes nonseparable in three degrees of freedom. The special case of perpendicular fields has been investigated both exper-

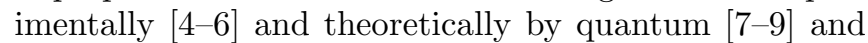
classical 10 13 methods. However, the case of arbitrary field orientation is the most general situation for atoms in uniform external fields, and therefore deserves appropriate attention. For the general field arrangement quantum calculations have been performed so far for weak external fields in first and second order perturbation theory [7]:8] and in the regime of very strong magnetic and electric fields 14.15.

In this Paper we investigate the hydrogen atom in external fields with arbitrary mutual orientations. In Sec. II we calculate the (numerically) exact quantum mechanical eigenenergies and oscillator strengths for a wide energy and field range, even far above the ionization threshold (Stark saddle point), by diagonalization of the Hamiltonian matrix in a complete set of eigenfunctions. On the basis of these exact quantum calculations we investigate the dependence of resonance energies on the angle between the external fields. At energies around the ionization threshold we observe large avoided crossings, which cannot be explained even qualitatively by conventional perturbation theory. These avoided crossings mainly occur between eigenstates of the same approximately conserved principal quantum number $n$. The phenomenon is interpreted as a quantum manifestation of intramanifold chaos, which has recently been discovered in classical investigations of the hydrogen atom in perpendicular crossed magnetic and electric fields [11].

To verify the interpretation of the avoided crossings as intramanifold chaos we extend, in Sec. III, the conventional perturbation theory [8] by taking into account all couplings between the angular momentum and the Runge-Lenz vector. This extended quantum mechanical perturbation theory can well reproduce, at least qualitatively, the large avoided crossings between levels of the same principal quantum number $n$.

Based on the Hamiltonian of the extended perturbation theory we analyze, in Sec. IV, the classical motion of the angular momentum and the Runge-Lenz vector by means of Poincaré surfaces of section (PSOS). The PSOS reveal large chaotic fractions of the classical phase space for those energy-field regions and mutual field orientations where the quantum spectra exhibit large avoided crossings. The classical calculations therefore confirm the interpretation of the intra $n$-manifold level repulsion as a quantum manifestation of intramanifold chaos.

In Sec. V we investigate the nearest-neighbor-spacing distributions of states with the same principal quantum number $n$. The distributions show a Poissonian behavior for parallel fields, where the intramanifold dynamics is regular. For arbitrarily oriented fields the distribution turns to a Brody-type distribution, indicating a mixed regular-chaotic intramanifold dynamics, in agreement with the classical Poincaré surface of section anal- 
ysis.

\section{EXACT QUANTUM CALCULATIONS}

The electronic motion of the hydrogen atom in uniform magnetic and electric fields with arbitrary mutual orientations is nonseparable in three degrees of freedom. The Hamiltonian [in atomic units, $f=F /\left(5.14 \times 10^{9} \mathrm{~V} / \mathrm{cm}\right)$, $\left.\gamma=B /\left(2.35 \times 10^{5} \mathrm{~T}\right)\right]$ reads

$$
H=\frac{1}{2} \mathbf{p}^{2}-\frac{1}{r}+\frac{1}{2} \gamma L_{z}+\frac{1}{8} \gamma^{2}\left(x^{2}+y^{2}\right)+f_{\perp} x+f_{\|} z,
$$

where the magnetic field is oriented in the $z$-direction and the electric field in the $(x, z)$-plane with $f_{\|}$and $f_{\perp}$ the components of the electric field parallel and perpendicular to the magnetic field axis, respectively. We neglect relativistic and spin effects as well as effects due to the finite nuclear mass, which yield only very small contributions in the energy-field regions we examine.

For the exact quantum calculations of the hydrogen atom in external fields with arbitrary mutual orientations we extend the method described in Ref. [9] for the special case of the hydrogen atom in perpendicular magnetic and electric fields $\left(f_{\|}=0\right)$. In perpendicular fields the parity with respect to the $(z=0)$-plane, $\pi_{z}$, is an exact quantum number and allows diagonalizing the Hamiltonian in each subspace $\pi_{z}= \pm 1$ separately. This symmetry is broken by the parallel component of the electric field resulting in an increase of the basis size for the numerical diagonalizations by about a factor of 2 .

For the numerical calculations we transform the Hamiltonian by introducing dilated semiparabolic coordinates

$$
\mu=\frac{1}{b} \sqrt{r+z}, \quad \nu=\frac{1}{b} \sqrt{r-z}, \quad \phi=\tan ^{-1} \frac{y}{x},
$$

where $b$ is a free length scale parameter. The Schrödinger equation in dilated semiparabolic coordinates then reads

$$
\begin{aligned}
& {\left[\triangle_{\mu}+\triangle_{\nu}-\left(\mu^{2}+\nu^{2}\right)+b^{4} \gamma\left(\mu^{2}+\nu^{2}\right) i \frac{\partial}{\partial \phi}\right.} \\
& -\frac{1}{4}\left(b^{4} \gamma\right)^{2} \mu^{2} \nu^{2}\left(\mu^{2}+\nu^{2}\right)-b^{6} f_{\|}\left(\mu^{4}-\nu^{4}\right) \\
& \left.-2 b^{6} f_{\perp} \mu \nu\left(\mu^{2}+\nu^{2}\right) \cos \phi+4 b^{2}\right] \Psi \\
= & \lambda\left(\mu^{2}+\nu^{2}\right) \Psi,
\end{aligned}
$$

with

$$
\triangle_{\rho}=\frac{\partial^{2}}{\partial \rho^{2}}+\frac{1}{\rho} \frac{\partial}{\partial \rho}+\frac{1}{\rho^{2}} \frac{\partial^{2}}{\partial \phi^{2}} ; \quad(\rho=\mu \text { or } \nu),
$$

and

$$
\lambda=-\left(1+2 b^{4} E\right)
$$

The Schrödinger equation (3) has the form of two coupled two-dimensional harmonic oscillators, and is therefore conveniently represented, in matrix form, in terms of the complete set of basis functions given by the product of the eigenstates $\left|N_{\rho} m\right\rangle$ of the two-dimensional harmonic oscillator, viz.

$$
\left|N_{\mu} N_{\nu} m\right\rangle=\left|N_{\mu} m\right\rangle \times\left|N_{\nu} m\right\rangle .
$$

One is led to a generalized eigenvalue problem with sparse symmetric matrices. The elements of these matrices can be calculated using the familiar operator relations for the two-dimensional harmonic oscillator. More details of the method are described in 16].

To account for continuum states we adopt the complexrotation method [17, 18], which is based on the replacement of the coordinate vector $\mathbf{r}$ with $\mathbf{r} e^{i \theta}$ in the Hamiltonian and the wave function, and has proved very efficient in the calculation of resonances above the ionization threshold for the hydrogen atom in magnetic fields 19 and in crossed magnetic and electric fields [9]. By this transformation, hidden resonances of the Hamiltonian in the continuum, associated with complex eigenvalues, are exposed, while the resonance wave functions can still be described by the $\mathcal{L}_{2}$ integrable basis functions (6), but with complex arguments. In our approach, the complex rotation by the angle $\theta$ corresponds to the complex dilatation

$$
b=|b| e^{i \frac{\theta}{2}}
$$

in (2) and in Schrödinger's equation (3).

Numerically a generalized eigenvalue problem with complex symmetric non-Hermitian matrices has to be solved, which was achieved by extending the spectral transformation Lanczos method [20 to complex matrices. From the imaginary part of the complex energies, $E$, we obtain the corresponding widths, $\Gamma$, and lifetimes, $T$, of the resonances by

$$
\Gamma=\frac{\hbar}{T}=-2 \operatorname{Im} E .
$$

As an example we calculated spectra of the hydrogen atom in combined magnetic and electric fields with $B=100 \mathrm{~T}, F=50 \mathrm{kV} / \mathrm{cm}$. These field strengths are by about an order of magnitude larger than typical laboratory fields. However, they allow studying the interesting physical effects at energy regions with a reasonably low density of states and reduce the computer resources (CPU time and storage requirements) necessary for the numerical calculation of the spectra. Bound states and resonances were obtained by numerical diagonalization of Eq. 3 in a basis set of dimension $\leq 6201$. The complex energies are presented in Fig. 1 for an angle $\beta=$ $\arctan \left(f_{\|} / f_{\perp}\right)=\pi / 4$ between the fields. The energy of the Stark saddle point, $E_{\mathrm{SP}}=-2 \sqrt{|\mathbf{f}|}$ au $=-1368 \mathrm{~cm}^{-1}$ is marked by an arrow in Fig. 1. Below the threshold all eigenvalues are located on the real axis (see Fig. 1). 


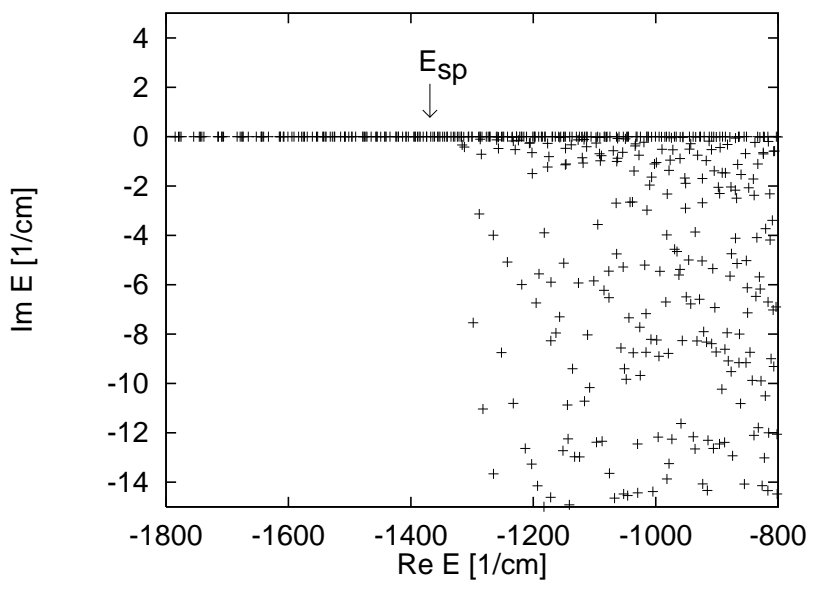

FIG. 1. Eigenenergies and resonances in the complex energy plane for the hydrogen atom in magnetic and electric fields $(B=100 \mathrm{~T}, F=50 \mathrm{kV} / \mathrm{cm})$ of mutual orientation $\beta=45^{\circ}$. The energy of the Stark saddle point is marked by an arrow.

Above threshold, long-lived states close to the real energy axis still exist, but hidden resonances of the Hamiltonian, associated with complex eigenvalues, are exposed by the complex rotation method.

With the energy eigenvalues and eigenvectors of (3) at hand it is also possible to calculate the cross section for dipole transitions from an initial state $\Psi_{0}$ with energy $E_{0}$. The cross section can be written in the form 21]

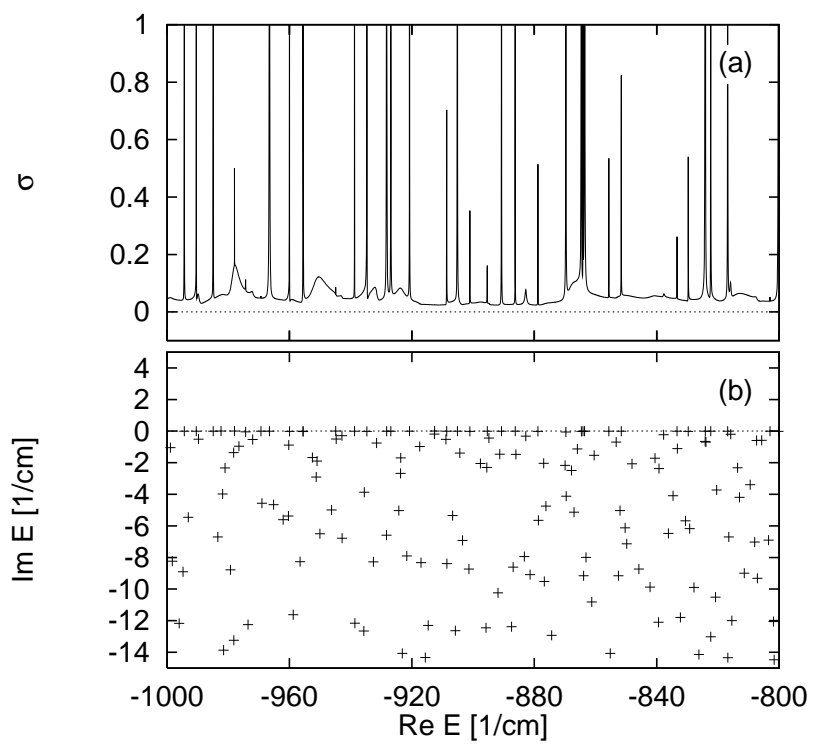

FIG. 2. (a) Photoabsorption spectrum for the hydrogen atom in magnetic and electric fields $(B=100 \mathrm{~T}$, $F=50 \mathrm{kV} / \mathrm{cm}$ ) of mutual orientation $\beta=45^{\circ}$. Transitions $|2 p 0\rangle \rightarrow\left|\psi_{f}\right\rangle$ with light polarized parallel to the magnetic field axis. (b) Resonances in the complex energy plane.

$$
\sigma(E)=4 \pi \alpha\left(E-E_{0}\right) \operatorname{Im}\left[\sum_{j} \frac{\left\langle\Psi_{0}|D| \Psi_{j}(\theta)\right\rangle^{2}}{E_{j}(\theta)-E}\right],
$$

where $\Psi_{j}(\theta)$ are final states at complex energies $E_{j}(\theta), D$ denotes the dipole operator for some given polarization, and $\alpha \approx 1 / 137$ is the fine-structure constant. As an example a photoabsorption spectrum for transitions from the initial state $|2 p 0\rangle$ with light polarized parallel to the magnetic field axis is presented in Fig. 2a. The chosen energy region is the high energy part of Fig. 1 and is well above the Stark saddle point, $E_{S P}=-1368 \mathrm{~cm}^{-1}$. The spectrum clearly exhibits sharp peaks of long-lived states superimposed on broad line shapes of rapidly decaying resonances and a continuous background. The complex energy eigenvalues are shown in Fig. $2 \mathrm{~b}$ for comparison.

For atoms in external fields with arbitrary mutual orientations the angle $\beta$ between the magnetic and electric field axis is an additional free parameter which allows one to continuously vary the geometry between the extreme situations of parallel and perpendicular fields. It is therefore interesting to fix the absolute values of the electric and magnetic field strength and to follow the energy levels as a function of the angle $\beta$. A part of such an $(E, \beta)$-diagram is shown in Fig. 3 at $B=100 \mathrm{~T}, F=50$ $\mathrm{kV} / \mathrm{cm}$. The dotted line marks the energy of the Stark saddle point. The $(E, \beta)$-diagram exhibits quite complicated patterns which cannot be explained, not even qualitatively, by conventional perturbation theory [8].

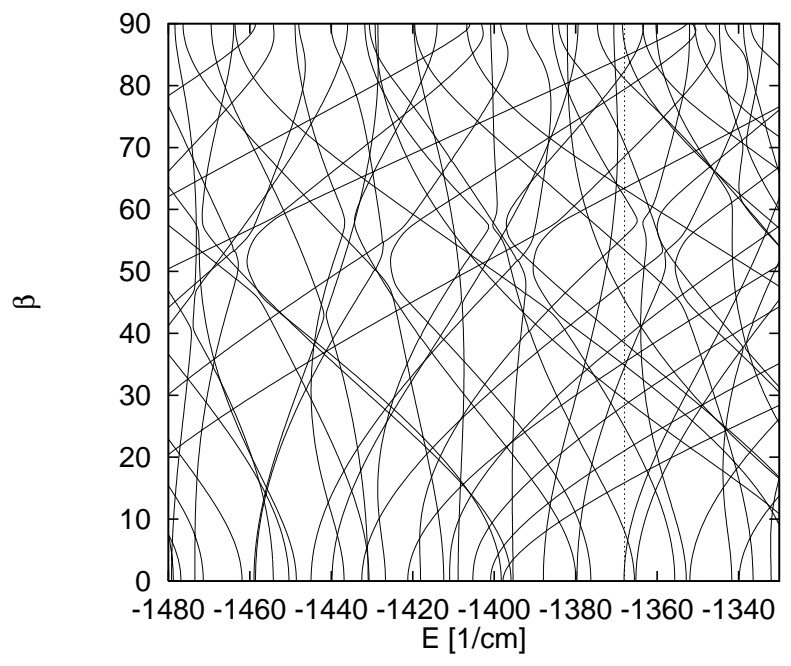

FIG. 3. Energy-angle diagram for the hydrogen atom in magnetic and electric fields at $B=100 \mathrm{~T}, F=50 \mathrm{kV} / \mathrm{cm} . \beta$ is the angle between the two field axis.

In particular, many levels undergo large avoided crossings, which are found to be most pronounced around angles $40^{\circ}<\beta<70^{\circ}$. The avoided crossings indicate chaos in the underlying classical dynamics. It is the purpose of the following Sections to investigate and analyze the patterns observed in Fig. 3 in more detail. This is performed by means of an extended quantum and classical pertur- 
bation theory, which will allow the interpretation of the avoided crossings as effects of intramanifold chaos.

\section{EXTENDED QUANTUM MECHANICAL PERTURBATION THEORY}

The perturbation theory of the hydrogen atom in combined electric and magnetic fields goes back to the early days of quantum mechanics. The first order perturbation theory was developed by Epstein [22], Born [23, and Pauli [7]. The second order equations describing the eigenenergies up to the quadratic terms in the field strengths were derived by Solov'ev [8]. The conventional perturbation theory is based classically on the construction of adiabatic constants of motion which are related to approximate quantum numbers in quantum mechanics. The results of second order perturbation theory agree well with our exact quantum calculations for weak external fields and at low excitation energies. However, at strong perturbations, e.g., at excitation energies around the Stark saddle point, the conventional perturbation theory cannot explain the breakdown of approximate quantum numbers and the onset of chaos in the classical dynamics. Therefore the large avoided crossings which have been observed, e.g., in Fig. 3 are not reproduced by the conventional perturbation theory. In the following we will briefly review the conventional perturbation theory and then generalize the method by taking into account all couplings between states within the same $n$-manifold up to second order in the external fields. The concept does not assume the existence of a complete set of approximate quantum numbers and therefore can describe and explain the phenomenon of intramanifold chaos.

The perturbation series for the hydrogen atom in external magnetic and electric fields up to second order in the field strengths reads

$$
H_{n}=-\frac{1}{2 n^{2}}+V_{1}+V_{2}+W
$$

with

$$
V_{1}=\frac{1}{2} \gamma L_{z}+\mathbf{f} \cdot \mathbf{r}
$$

the paramagnetic and linear Stark terms,

$$
V_{2}=\frac{1}{8} \gamma^{2} \rho^{2}
$$

the diamagnetic term, and

$$
W=(\mathbf{f} \cdot \mathbf{r}) G_{n}(\mathbf{f} \cdot \mathbf{r})
$$

the quadratic Stark effect, with $G_{n}$ the Green's function of the field free hydrogen atom, $H_{0}$. The operators $V_{1}$, $V_{2}$, and $W$ can now be replaced within each $n$-manifold by operator identities in terms of the angular momentum

$$
\mathbf{L}=\mathbf{r} \times \mathbf{p}
$$

and the Runge-Lenz vector

$$
\mathbf{A}=\frac{1}{\sqrt{-2 H_{0}}}\left[\frac{1}{2}(\mathbf{p} \times \mathbf{L}-\mathbf{L} \times \mathbf{p})-\frac{\mathbf{r}}{r}\right]
$$

or in terms of the linear combinations of these vectors

$$
\mathbf{I}_{1,2}=\frac{1}{2}(\mathbf{L} \pm \mathbf{A}) .
$$

The vectors $\mathbf{I}_{1}$ and $\mathbf{I}_{2}$ commute with each other and their components obey the commutation relations for angular momentum operators,

$$
\begin{aligned}
& {\left[I_{1 j}, I_{2 k}\right]=0,} \\
& {\left[I_{1 j}, I_{1 k}\right]=i \epsilon_{j k l} I_{1 l},} \\
& {\left[I_{2 j}, I_{2 k}\right]=i \epsilon_{j k l} I_{2 l} .}
\end{aligned}
$$

Both vectors have the same norm, which depends on the principal quantum number,

$$
\left|\mathbf{I}_{1}\right|=\left|\mathbf{I}_{2}\right|=\frac{1}{2} \sqrt{n^{2}-1} .
$$

The operator identity for $V_{1}$ in a given $n$-manifold then reads [7]

$$
V_{1}=\vec{\omega}_{1} \cdot \mathbf{I}_{1}+\vec{\omega}_{2} \cdot \mathbf{I}_{2}
$$

with

$$
\vec{\omega}_{1,2}=\frac{1}{2}(\vec{\gamma} \mp 3 n \vec{f})
$$

as is illustrated in Fig. 4. Because $\mathbf{I}_{1}$ and $\mathbf{I}_{2}$ independently fulfill the commutation algebra of two angular momenta, the operator $V_{1}$ in (19) can be quantized immediately to obtain the energy correction in first order perturbation theory

$$
E_{n n^{\prime} n^{\prime \prime}}^{(1)}=\omega_{1} n^{\prime}+\omega_{2} n^{\prime \prime}
$$

with $\omega_{1,2}=\left|\vec{\omega}_{1,2}\right|$ and quantum numbers $n^{\prime}, n^{\prime \prime}=-(n-$ $1) / 2,-(n-3) / 2, \ldots,+(n-1) / 2$. Classically, $\mathbf{I}_{1}$ and $\mathbf{I}_{2}$ turn around the vectors $\vec{\omega}_{1}$ and $\vec{\omega}_{2}$, whose absolute values are the frequencies of the secular motion (see Fig. 4).

The operator identities for $V_{2}$ and $W$ are the starting point for the second order perturbation theory and have been derived by Solov'ev [8],

$$
\begin{aligned}
& V_{2}=\frac{n^{2} \gamma^{2}}{16}\left(n^{2}+3+L_{z}^{2}+4 \mathbf{A}^{2}-5 A_{z}^{2}\right), \\
& W=-\frac{n^{4} f^{2}}{16}\left(5 n^{2}+31+24 \mathbf{L}^{2}-21 L_{f}^{2}+9 A_{f}^{2}\right),
\end{aligned}
$$

with $L_{f}$ and $A_{f}$ the projections of the angular momentum and Runge-Lenz vector on the electric field axis.

Except for the special case of perpendicular fields where we have $\omega_{1}=\omega_{2}=\frac{1}{2} \sqrt{\gamma^{2}+9 n^{2} f^{2}}$, the degeneracy of the $n^{2}$ states belonging to each $n$-manifold is already completely destroyed in first order perturbation 


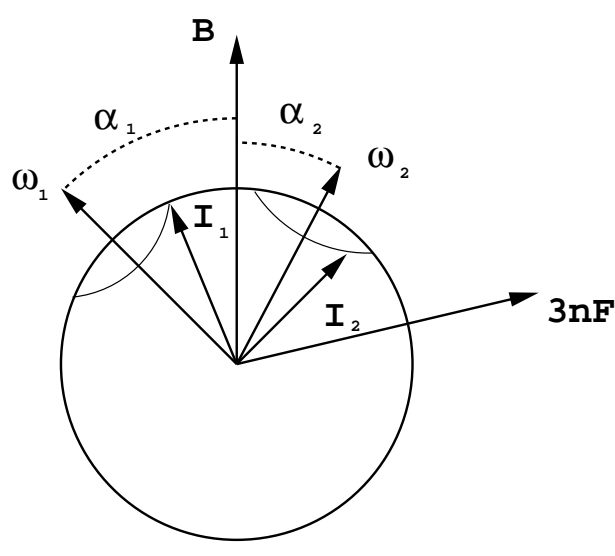

FIG. 4. Schematic view of the vectors $\vec{\omega}_{1}, \vec{\omega}_{2}$ and $\mathbf{I}_{1}, \mathbf{I}_{2}$ which are used in perturbation theory.

theory. In conventional perturbation theory, the second order energy correction, $E_{n n^{\prime} n^{\prime \prime}}^{(2)}$ is given as the expectation value of $V_{2}+W$ in the eigenstate $\left|n n^{\prime} n^{\prime \prime}\right\rangle$,

$$
\begin{aligned}
& E_{n n^{\prime} n^{\prime \prime}}^{(2)}=\left\langle n n^{\prime} n^{\prime \prime}\left|V_{2}+W\right| n n^{\prime} n^{\prime \prime}\right\rangle \\
= & -\frac{n^{4} f^{2}}{16}\left[17 n^{2}+19-12\left(n^{\prime 2}+n^{\prime} n^{\prime \prime} \cos \left(\alpha_{1}+\alpha_{2}\right)\right.\right. \\
& \left.\left.+n^{\prime \prime 2}\right)\right]+\frac{n^{2} \gamma^{2}}{48}\left[7 n^{2}+5+4 n^{\prime} n^{\prime \prime} \sin \alpha_{1} \sin \alpha_{2}\right. \\
& +\left(n^{2}-1\right)\left(\cos ^{2} \alpha_{1}+\cos ^{2} \alpha_{2}\right)-12\left(n^{\prime 2} \cos ^{2} \alpha_{1}\right. \\
& \left.\left.-n^{\prime} n^{\prime \prime} \cos \alpha_{1} \cos \alpha_{2}+n^{\prime \prime 2} \cos ^{2} \alpha_{2}\right)\right],
\end{aligned}
$$

where $\alpha_{1}$ and $\alpha_{2}$ are the angles between the magnetic field axis and the vectors $\vec{\omega}_{1}$ and $\vec{\omega}_{2}$, respectively (see Fig. 4). The energy eigenvalues are finally obtained as $E_{n n^{\prime} n^{\prime \prime}}=-1 / 2 n^{2}+E_{n n^{\prime} n^{\prime \prime}}^{(1)}+E_{n n^{\prime} n^{\prime \prime}}^{(2)}$. Note that Eq. 24 is not valid for perpendicular fields because in this case the degeneracy of the $n$-manifolds is not completely destroyed in first order perturbation theory [8,24]. However, the qualitative results of the following discussion are not changed when the conventional perturbation theory for nearly perpendicular fields is applied instead of Eq. 24 [16].

The results of the conventional second order perturbation theory for non-perpendicular fields are compared to the exact quantum calculations in Fig. 5. The dashed lines in Figs. 5a and 5b are the levels of manifolds $n=9$ and $n=10$, respectively. To simplify the identification the exact levels with the corresponding principal quantum numbers are highlighted by solid lines while other levels are drawn by dotted lines. Although the identification is possible, the comparison reveals remarkable discrepancies between the results of conventional perturbation theory and the exact quantum results in this energy regime. In the conventional perturbation theory all level crossings are exact and cannot reproduce the large avoided crossings discussed above. Another interesting

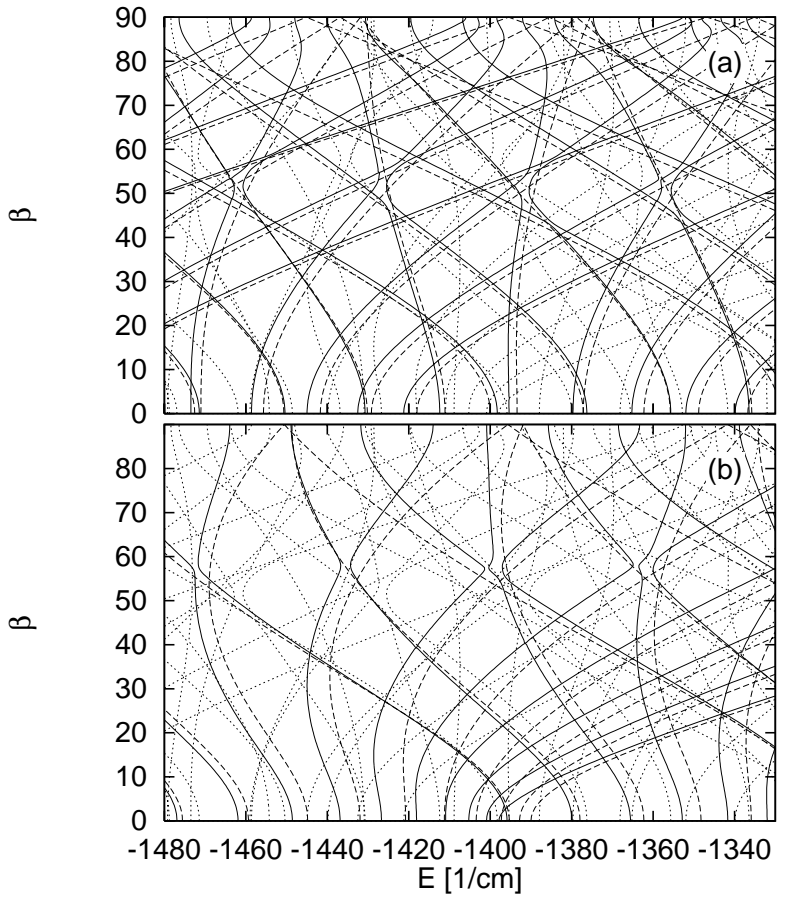

FIG. 5. Exact energy eigenvalues (solid lines) compared with energy values calculated by conventional second order perturbation theory (dashed lines). (a) Principal quantum number $n=9$; (b) $n=10$.

result of the comparisons in Fig. 5 is that the large avoided crossings do not occur between levels with different principal quantum numbers. Therefore they cannot be interpreted as an $n$-mixing effect, which has been identified as the origin of level repulsion in the hydrogen atom in magnetic or parallel magnetic and electric fields. The level repulsions in Fig. 5 solely occur between levels of the same $n$-manifold and therefore must be interpreted as a mixing between states $\left|n n^{\prime} n^{\prime \prime}\right\rangle$ with different quantum numbers $n^{\prime}$ and $n^{\prime \prime}$ but with the same principal quantum number $n$. The breakdown of the quantum numbers $n^{\prime}$ and $n^{\prime \prime}$ is classically related to intramanifold chaos, which will be discussed in Sec. IV.

We now demonstrate that the avoided crossings are an effect of intramanifold mixing of states. The conventional perturbation theory is based on the assumption that the external fields are weak, i.e., the linear terms in the external fields, $V_{1}$, and the quadratic terms, $V_{2}+W$, can be treated separately as first and second order perturbations. We now extend the conventional perturbation theory by diagonalizing the complete perturbation operator $V_{1}+V_{2}+W$ in Eq. 10 within a given $n$-manifold. As a basis set we choose the parabolic states, i.e., simultaneous eigenstates of the Coulomb Hamiltonian, $H_{0}$, and the $z$ components of the angular momentum and RungeLenz vectors, $L_{z}$ and $A_{z}$. By substituting the angular momentum and Runge-Lenz vector in Eqs. 19, 22, and 23 with $\mathbf{L}=\mathbf{I}_{1}+\mathbf{I}_{2}$ and $\mathbf{A}=\mathbf{I}_{1}-\mathbf{I}_{2}$ and applying 
the commutator algebra for the operators $\mathbf{I}_{1}$ and $\mathbf{I}_{2}$ it is a straightforward task to compute the required matrix elements of $V_{1}+V_{2}+W$. The energy eigenvalues are obtained by numerical diagonalization of the perturbation matrix of dimension $n^{2} \times n^{2}$.

The results of the extended perturbation theory are presented in Fig. 6 for parts of the manifolds $n=9$ and $n=10$. The exact quantum levels (highlighted solid and dotted lines) are the same as in Fig. 5.

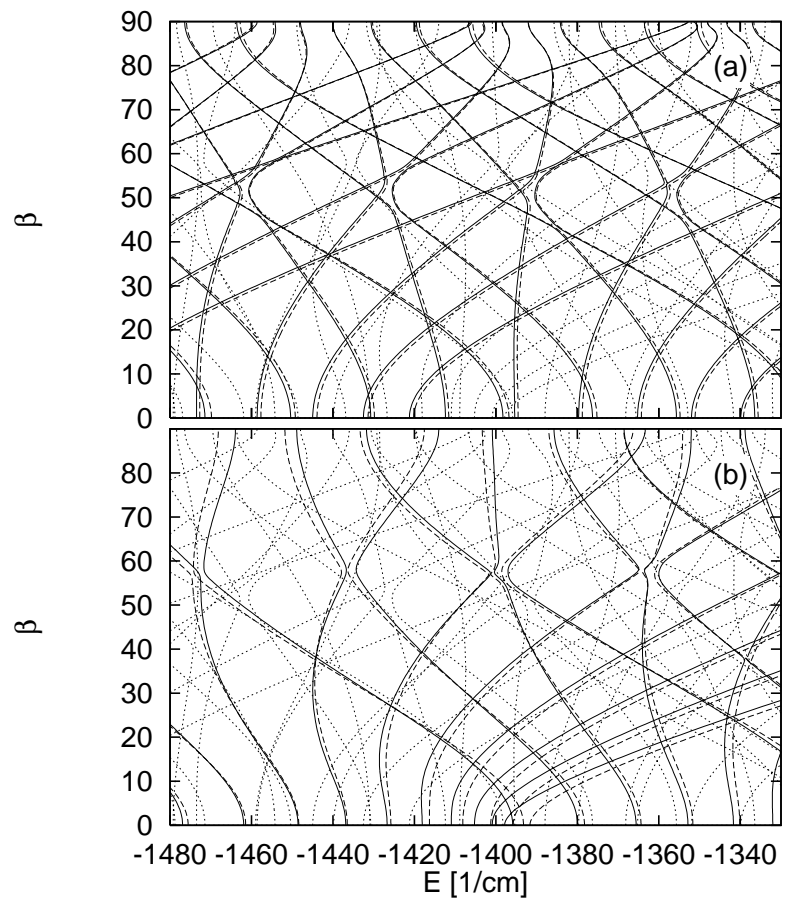

FIG. 6. Same as Fig. 5 but for the comparison between the exact eigenenergies (solid lines) and the results of extended perturbation theory (dashed lines). (a) $n=9$; (b) $n=10$.

The dashed lines are the eigenvalues from the diagonalization of the complete perturbation matrix (10). They do not totally agree with the exact levels, but this cannot be expected at the high excitation energies close to the Stark saddle point. However, Fig. 6 qualitatively exhibits the same level dynamics for both the solid and dashed lines for all mutual field orientations, i.e., from parallel fields $(\beta=0)$ to perpendicular fields $(\beta=\pi / 2)$. In particular, the avoided crossings between levels of the same $n$-manifold are well reproduced within the extended perturbation theory, and this strongly supports the interpretation of the observed level repulsion as an effect of intramanifold mixing. The $n$-mixing of states will become important only at higher energies far above the ionization threshold.

\section{CLASSICAL INTRAMANIFOLD DYNAMICS}

The hydrogen atom in magnetic and electric fields with arbitrary mutual orientations is nonintegrable in three degrees of freedom. However, for weak external fields and low excitation energies three approximate quantum numbers $n, n^{\prime}$, and $n^{\prime \prime}$ exist. In classical mechanics these quantum numbers are related to three constants of the motion, viz. the length of the vectors $\mathbf{I}_{1}$ and $\mathbf{I}_{2}$,

$$
\left|\mathbf{I}_{1}\right|=\left|\mathbf{I}_{2}\right|=\frac{1}{2} n
$$

(note the slight difference to the quantum expression (18)) and their projections on the axis $\vec{\omega}_{1}$ and $\vec{\omega}_{2}$ defined in Eq. 20. $\mathbf{I}_{1}$ and $\mathbf{I}_{2}$ turn around the vectors $\vec{\omega}_{1}$ and $\vec{\omega}_{2}$ with constant frequencies (see Fig. 4), i.e., the classical dynamics is completely regular. In the extended perturbation theory discussed in the previous Section, states $\left|n n^{\prime} n^{\prime \prime}\right\rangle$ with the same principle quantum number $n$ but with different quantum numbers $n^{\prime}$ and $n^{\prime \prime}$ are mixed, i.e., two approximate quantum numbers are destroyed. The breakdown of two constants of motion can lead to a chaotic secular motion of the vectors $\mathbf{I}_{1}$ and $\mathbf{I}_{2}$ even when the principal quantum number, $n$, is still conserved. This phenomenon is called intramanifold chaos and has been discovered in the hydrogen atom in perpendicular crossed magnetic and electric fields [11]. Here we study the classical intramanifold dynamics of the hydrogen atom in external fields with arbitrary mutual orientations, and are especially interested in the dependence of the classical dynamics on the angle between the external fields. The quantum calculations in Sec. III revealed large avoided crossings at angles $40^{\circ}<\beta<70^{\circ}$. If the level repulsion is the quantum counterpart of classical intramanifold chaos, the classical dynamics should be particularly irregular at those field orientations.

To investigate the intramanifold dynamics we solve the equations of motion for the secular motion of the two vectors $\mathbf{I}_{1}$ and $\mathbf{I}_{2}$ defined in (16). By expressing the angular momentum and Runge-Lenz vector in the Hamiltonian (10) in terms of $\mathbf{I}_{1}$ and $\mathbf{I}_{2}$, scaled with the principal quantum number, viz. $\mathbf{I}_{1,2} \rightarrow \mathbf{I}_{1,2} / n$, we obtain the classical Hamiltonian valid within a given $n$-manifold,

$$
\begin{aligned}
& \mathcal{H} \equiv 2 n^{2} H_{n}=2 n^{2} E \\
= & -1+n^{3} \gamma\left(I_{1 z}+I_{2 z}\right) \\
- & 3 n^{4} f\left[\sin \beta\left(I_{1 x}-I_{2 x}\right)+\cos \beta\left(I_{1 z}-I_{2 z}\right)\right] \\
+ & \frac{1}{8}\left(n^{3} \gamma\right)^{2}\left[3-4\left(I_{1 z}^{2}+I_{2 z}^{2}-I_{1 z} I_{2 z}\right)-8 I_{1 x} I_{2 x}-8 I_{1 y} I_{2 y}\right] \\
- & \frac{1}{8}\left(n^{4} f\right)^{2}\left\{17-12 \sin ^{2} \beta\left(I_{1 x}^{2}+I_{2 x}^{2}\right)-12 \cos ^{2} \beta\left(I_{1 z}^{2}+I_{2 z}^{2}\right)\right. \\
+ & \left(48-60 \sin ^{2} \beta\right) I_{1 x} I_{2 x}+48 I_{1 y} I_{2 y}+\left(48-60 \cos ^{2} \beta\right) I_{1 z} I_{2 z} \\
- & \left.12 \sin \beta \cos \beta\left[5\left(I_{1 x} I_{2 z}+I_{1 z} I_{2 x}\right)+2\left(I_{1 x} I_{1 z}+I_{2 x} I_{2 x}\right)\right]\right\} .
\end{aligned}
$$

The classical equations of motions are now obtained from the Hamiltonian (26) by the Poisson brackets

$$
\begin{aligned}
& \dot{\mathbf{I}}_{1}=\left[\mathbf{I}_{1}, \mathcal{H}\right], \\
& \dot{\mathbf{I}}_{2}=\left[\mathbf{I}_{2}, \mathcal{H}\right],
\end{aligned}
$$


which can be solved with the help of the elementary Poisson brackets for the components of $\mathbf{I}_{1}$ and $\mathbf{I}_{2}$,

$$
\begin{aligned}
& {\left[I_{1 j}, I_{2 k}\right]=0,} \\
& {\left[I_{1 j}, I_{1 k}\right]=\epsilon_{j k l} I_{1 l},} \\
& {\left[I_{2 j}, I_{2 k}\right]=\epsilon_{j k l} I_{2 l} .}
\end{aligned}
$$

The classical intramanifold dynamics depends on the energy and field strengths scaled with the principal quantum number, $n^{2} E, n^{3} \gamma$, and $n^{4} f$, and on the angle, $\beta$, between the fields. The classical trajectories are computed in the six-dimensional space $\left\{\mathbf{I}_{1}(t), \mathbf{I}_{2}(t)\right\}$. However, the norm of $\mathbf{I}_{1}$ and $\mathbf{I}_{2}$ is conserved (25), and therefore the effective phase space is four-dimensional. A convenient parameterization of the four-dimensional phase space are the projections of $\mathbf{I}_{1}$ and $\mathbf{I}_{2}$ on the axis $\vec{\omega}_{1}$ and $\vec{\omega}_{2}$ (see Fig. 4), called $J_{1 z}$ and $J_{2 z}$ in what follows, and the polar angles $\phi_{1}$ and $\phi_{2}$ of $\mathbf{I}_{1}$ and $\mathbf{I}_{2}$ with respect to the $\vec{\omega}_{1,2}$ axis. Note that $\left\{J_{1 z}, \phi_{1}\right\}$ and $\left\{J_{2 z}, \phi_{2}\right\}$ are action-angle variables and $J_{1 z}$ and $J_{2 z}$ are constants of motion in the limit of weak non-perpendicular external fields.

A common way of visualizing the classical dynamics of a system is the method of Poincare surfaces of section (PSOS). In a system with $n$ degrees of freedom the PSOS is in general a $(2 n-2)$-dimensional subspace of the $(2 n)$ dimensional phase space. Elliptic fixpoints surrounded by torus structures indicate regular classical dynamics while chaotic motion is indicated by stochastic layers in the PSOS. For the analysis of the intramanifold dynamics we use the action-angle variable of the weak field limit and define the intersections with the PSOS by $\phi_{2}=0$. The two-dimensional PSOS is now defined by the doublet $\left\{J_{1 z}, \phi_{1}\right\}$, while $J_{2 z}$ is given implicitly via the conservation of energy.

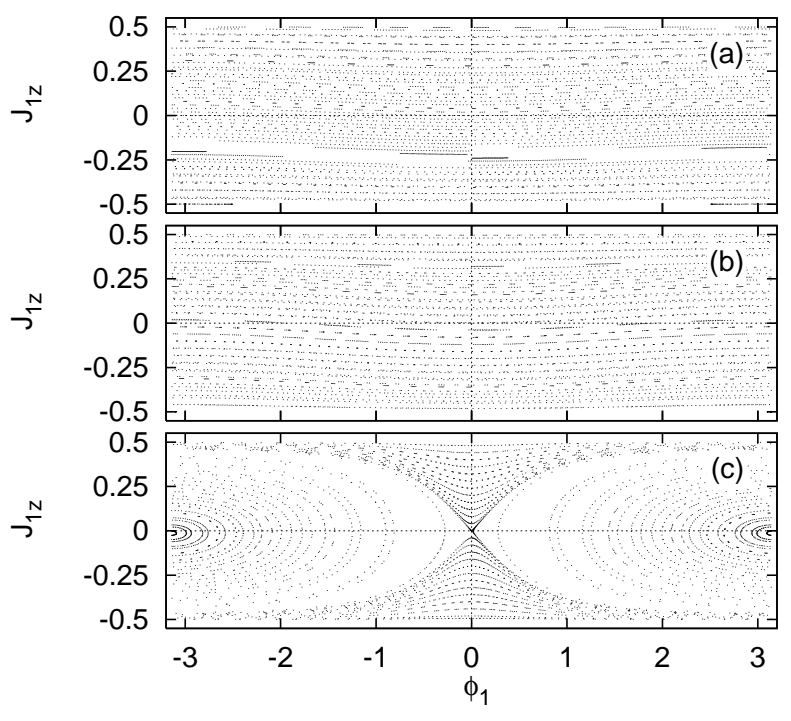

FIG. 7. Poincaré surfaces of section for the classical intramanifold dynamics with parameters $n^{3} \gamma=0.053$, $n^{4} f=0.0061, n^{2} E=-0.5$. Mutual field orientation (a) $\beta=0^{\circ}$, (b) $\beta=45^{\circ}$, (c) $\beta=90^{\circ}$.
As an example we calculated PSOS for very weak external fields, $n^{3} \gamma=0.053, n^{4} f=0.0061$, which is related to the manifold $n=5$ at the field strengths $B=100$ T, $F=50 \mathrm{kV} / \mathrm{cm}$ chosen for the quantum calculations in Secs. II and III. Figs. 7a and 7b present the results for mutual field orientations $\beta=0$ (parallel fields) and $\beta=\pi / 4$, respectively. Qualitatively both PSOS look the same, i.e., they exhibit nearly parallel lines with $J_{1 z} \approx$ const. This is a verification of the fact that $J_{1 z}$ is a constant of motion in the limit of weak external nonparallel fields. For the special case of perpendicular fields $(\beta=\pi / 2)$ the PSOS have a qualitatively different appearance (Fig. 7c). This is a consequence of the fact that in perpendicular fields the degeneracy is not completely destroyed in first order perturbation theory $\left(\omega_{1}=\omega_{2}\right.$ in Eq. 21), and therefore $J_{1 z}$ is no constant of motion. However, Fig. 7 clearly reveals that the classical intramanifold dynamics is completely regular in the weak field limit for all mutual field orientations.

The situation changes when we look at the PSOS for stronger interaction of the hydrogen atom with the external fields. To search for classical intramanifold chaos we analyzed the dynamics at parameters $n^{3} \gamma=0.74$, $n^{4} f=0.2, n^{2} E=-0.5$, which is related to the manifold

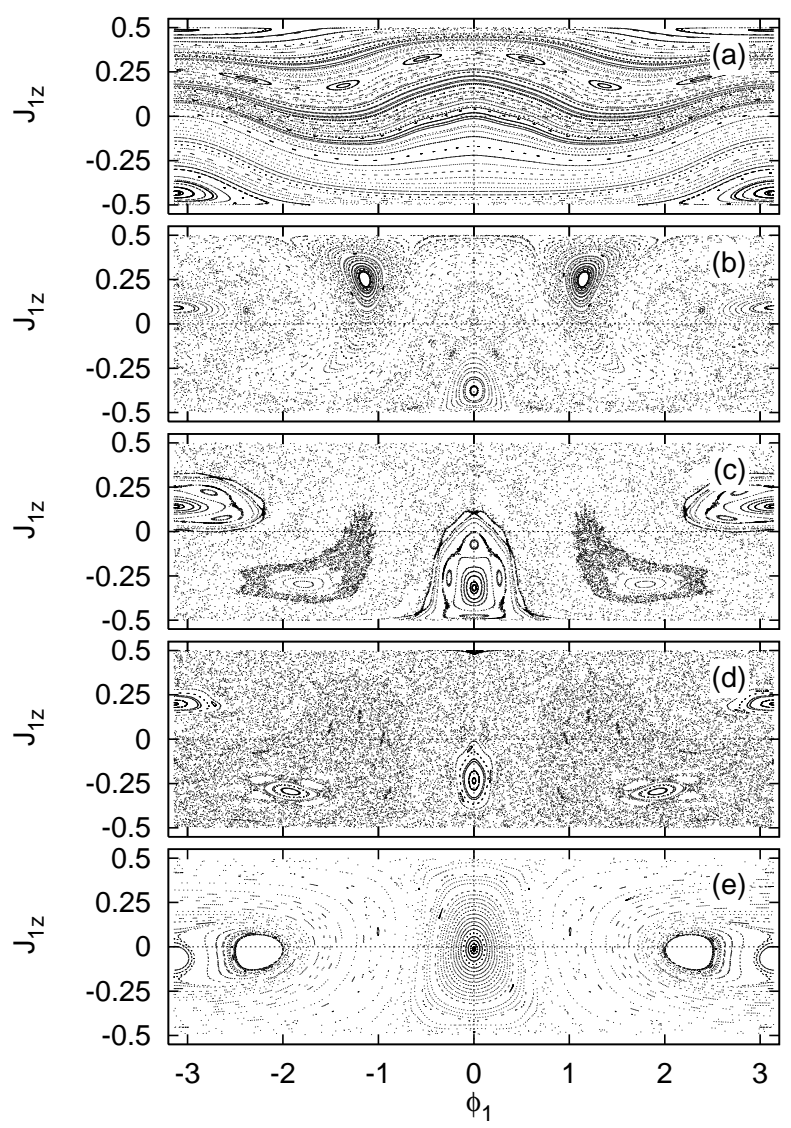

FIG. 8. Same as Fig. 7 but with parameters $n^{3} \gamma=0.74$, $n^{4} f=0.2, n^{2} E=-0.5$ and mutual field orientation (a) $\beta=20^{\circ}$, (b) $\beta=58^{\circ}$, (c) $\beta=60^{\circ}$, (d) $\beta=62^{\circ}$, (e) $\beta=90^{\circ}$. 
$n=12$ at the field strengths $B=100 \mathrm{~T}, F=50 \mathrm{kV} / \mathrm{cm}$. The Poincaré surfaces of section are presented in Fig. 8 for various mutual orientations of the external fields. In the region $0 \leq \beta<40^{\circ}$ the torus structures $J_{1 z} \approx$ const observed at weak fields (Figs. 7a and 7b) are strongly distorted (see Fig. $8 \mathrm{a}$ at $\beta=20^{\circ}$ ). However, the tori are not destroyed, i.e., the classical intramanifold dynamics is mostly regular apart from tiny stochastic regions around hyperbolic fix points. Note that no intramanifold level repulsion was observed for those field orientations in the quantum calculations of Secs. II and III.

The onset of strong classical intramanifold chaos is discovered at mutual field orientations $40^{\circ}<\beta<70^{\circ}$. Examples at $\beta=58^{\circ}, \beta=60^{\circ}$, and $\beta=62^{\circ}$, are given in Figs. 8b, 8c, and 8d, respectively. They clearly exhibit mixed regular-chaotic classical dynamics with large or even dominating stochastic, i.e., chaotic phase space regions. Fig. 8 also shows a sensitive dependence of the classical dynamics on the angle between the external fields. Even small deviations $\Delta \beta=2^{\circ}$ of the mutual field orientation result in significant changes of the PSOS. For example at $\beta=58^{\circ}$ (Fig. 8b) there exists a regular region at the top of the PSOS, which vanishes at $\beta=60^{\circ}$ in Fig. 8c. At that angle the PSOS exhibits two large irregular areas which are dynamically separated. The dynamical barrier is resolved in Fig. 8d where only a few stability islands are embedded in one large irregular region. The field arrangement where strong classical intramanifold chaos is observed $\left(40^{\circ}<\beta<70^{\circ}\right)$ coincides with the region where large avoided crossings between quantum levels with the same principal quantum number, $n$, were found in Fig. 6. This strongly supports the interpretation of the level repulsion as a quantum manifestation of intramanifold chaos.

If the angle between the fields is further increased $\left(\beta>70^{\circ}\right)$ the intramanifold dynamics becomes more and more regular again. The PSOS for the special case of perpendicular fields $\left(\beta=90^{\circ}\right)$ is presented in Fig. 8e. Chaotic regions exist in agreement with previous classical investigations of the crossed field atom [1], however, the PSOS is clearly dominated by regular torus structures.

\section{INTRAMANIFOLD LEVEL STATISTICS}

It is well established that the nearest-neighbor-spacing distribution (NNS) of integrable quantum systems is given, after unfolding the spectra to unit mean level spacing $\langle s\rangle=1$, by a Poisson distribution

$$
P_{\text {Poisson }}(s)=\exp [-s],
$$

while quantum systems with a fully chaotic (ergodic) underlying classical dynamics are characterized by the Wigner distribution

$$
P_{\text {Wigner }}(s)=\frac{\pi s}{2} \exp \left[-\frac{\pi}{4} s^{2}\right]
$$

obtained from random matrix theory [25,26]. In systems with a mixed regular-chaotic classical dynamics the nearest-neighbor-spacing distribution can be phenomenologically described by the Brody distribution 27]

$$
P_{\text {Brody }}(s ; q)=(q+1) \alpha s^{q} \exp \left[-\alpha s^{q+1}\right]
$$

where

$$
\alpha=\left[\Gamma\left(\frac{q+2}{q+1}\right)\right]^{q+1}
$$

and $q$ is a parameter which interpolates between the Poisson distribution $(q=0)$ and the Wigner distribution $(q=1)$ and is roughly related to the percentage of chaotic phase space volume of the underlying classical system.

Here we do not intend to compare nearestneighbor-spacing distributions with the complete threedimensional classical dynamics of the hydrogen atom in external magnetic and electric fields with arbitrary mutual orientations. Instead, we want to search for fingerprints of intramanifold quantum chaos in the level statistics. We therefore do not investigate the NNS distribution of all levels of a given spectrum but analyze separately the spacings of neighboring states with the same principal quantum number, $n$. For direct comparisons with the PSOS of the classical intramanifold dynamics (see Sec. IV) the spacing distributions should be obtained at constant parameters $n^{3} \gamma, n^{4} f$, and $n^{2} E$ for the scaled field strengths and energy. In order to get a reliable statistics for the problem we have to calculate a sufficiently large set of eigenvalues. To fulfill these conditions we calculated eigenenergies at constant scaled field strengths $n^{3} \gamma$ and $n^{4} f$ by means of the extended second order perturbation theory derived in Sec. III. The Hamiltonian (10) was diagonalized numerically for all $n$ manifolds from $n=50$ to $n=60$. The resulting spectra were unfolded separately to mean level spacing $\langle s\rangle=1$ [25]. For the NNS distributions we included only eigenstates whose scaled energies deviate by at most $0.1 n^{3} \gamma$ from the given value of $n^{2} E$. This ensures that the scaled energy is approximately constant and the NNS distributions can be compared to the classical dynamics, viz. the PSOS, at a given constant energy.

As an example, Fig. 9 presents the NNS distribution at field parameters $n^{3} \gamma=0.31, n^{4} f=0.064, \beta=45^{\circ}$, and for approximately constant scaled energy $n^{2} E \approx-0.5$. These parameters represent the situation of the manifold $n=9$ at $B=100 \mathrm{~T}$ and $F=50 \mathrm{kV} / \mathrm{cm}$ in Fig. 6a. However, to increase the density of states for a more reliable statistics we analyzed the manifolds $n=50$ to $n=60$ at correspondingly rescaled energies and field strengths. The histogram in Fig. 9 is close to a Poisson distribution (29) indicating a regular intramanifold dynamics. An even better fit is obtained by a Brody distribution (31) with $q=0.08$ (dashed line in Fig. 9). The deviations from the Poisson distribution can be interpreted as the onset of intramanifold quantum chaos. The decrease of 


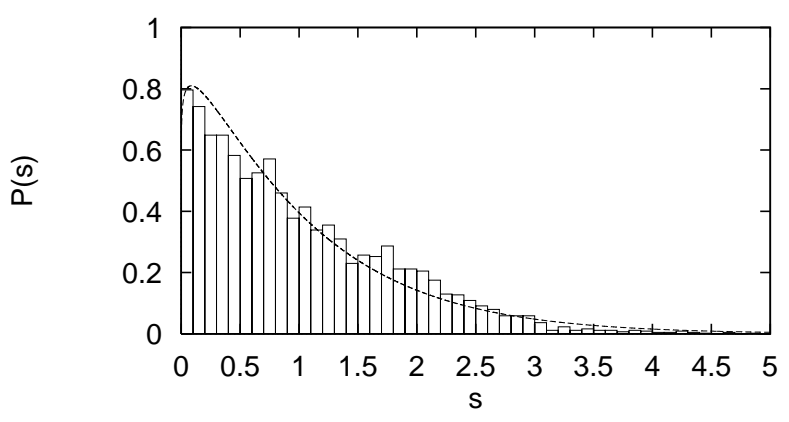

FIG. 9. Intramanifold nearest-neighbor-spacing distribution for the parameters $n^{3} \gamma=0.31, n^{4} f=0.064, n^{2} E \approx-0.5$, $\beta=45^{\circ}$. The dashed line is a Brody distribution with $q=0.08$.

the probability $P(s)$ at small spacings $s$ is related to the observation of level repulsions in Fig. 6a. For a more pronounced verification of intramanifold quantum chaos in NNS distributions we investigated quantum spectra at parameters $n^{3} \gamma=0.74, n^{4} f=0.2, n^{2} E \approx-0.5$, i.e., the same parameters as for the analysis of the classical intramanifold dynamics in Fig. 8 of Sec. IV. The NNS distributions for the same set of five different mutual field orientations between $\beta=20^{\circ}$ and $\beta=90^{\circ}$ are presented in Fig. 10. The histograms indicate a predominately regular intramanifold dynamics at $\beta=20^{\circ}$ (Fig. 10a) and $\beta=90^{\circ}$ (Fig. 10e) with Brody parameters $q=0.1$ and $q=0.05$, respectively, and this is in excellent agreement with the regular torus structures in the PSOS of Figs. 8a and 8e. The NNS distribution changes dramatically at mutual field orientations around $\beta=60^{\circ}$ (see Figs. 10b - 10d). The histograms turn into Brody distributions with much higher Brody parameters, up to $q=0.45$, indicating a large irregular part of the underlying classical intramanifold dynamics. Again the behavior of the NNS distributions is in very good agreement with the PSOS of Figs. $8 \mathrm{~b}-8 \mathrm{~d}$, which exhibit large stochastic regions at field orientations around $\beta=60^{\circ}$. Another interesting aspect which can be observed in Fig. 10 is the sensitive dependence of the NNS distributions on small variations of the mutual orientation between the external fields around $\beta \approx 60^{\circ}$. The Brody parameter increases from $q=0.3$ at $\beta=58^{\circ}$ (Fig. 10b) to $q=0.45$ at $\beta=60^{\circ}$ (Fig. 10c) and then decreases to $q=0.2$ at $\beta=62^{\circ}$ (Fig. 10d). The rapid changes of the Brody parameter reflect the sensitive dependence of the classical intramanifold dynamics on the field orientation discovered in the PSOS (see Figs. 8b-8d).

\section{CONCLUSION}

We have investigated the hydrogen atom in electric and magnetic fields with arbitrary mutual orientations by means of exact quantum calculations, an extended

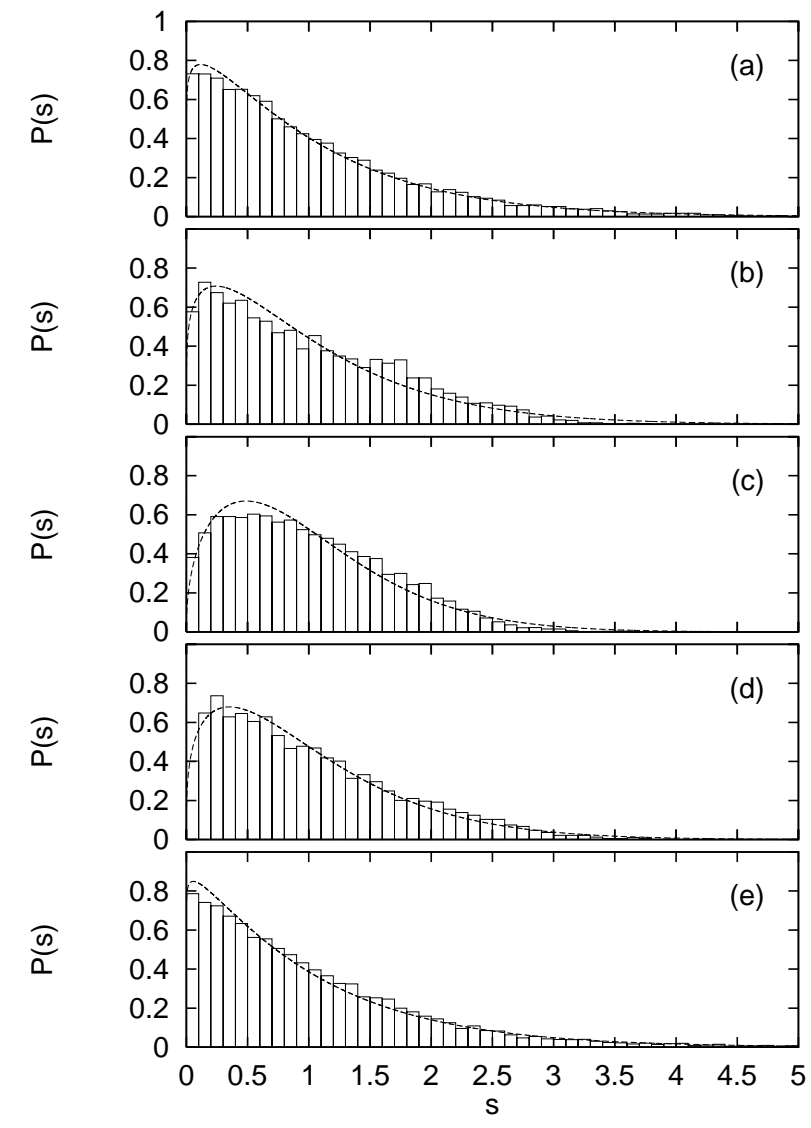

FIG. 10. Intramanifold nearest-neighbor-spacing distributions for the parameters $n^{3} \gamma=0.74, n^{4} f=0.2, n^{2} E \approx-0.5$ and mutual field orientations (a) $\beta=20^{\circ}$, (b) $\beta=58^{\circ}$, (c) $\beta=60^{\circ}$, (d) $\beta=62^{\circ}$, (e) $\beta=90^{\circ}$. The dashed lines are fitted Brody distributions with (a) $q=0.1$, (b) $q=0.2$, (c) $q=0.45$, (d) $q=0.3$, (e) $q=0.05$.

second order perturbation theory, classical Poincaré surface of section analysis of the intramanifold dynamics, and statistical analysis of the intramanifold nearestneighbor-spacing distributions. For mutual orientations around $\beta \approx 60^{\circ}$ and excitation energies around the Stark saddle point the exact quantum calculation reveal large avoided crossings between states with the same approximate principal quantum number, $n$. Because the level repulsion occurs preferably within a given $n$-manifold it is interpreted as a quantum manifestation of intramanifold chaos. This interpretation is strongly supported by the analysis of both the classical intramanifold dynamics and the intramanifold NNS distributions. The classical analysis exhibits large stochastic regions, i.e., chaotic motion in the PSOS at those parameters where strong level repulsion has been observed in the quantum spectra, and the intramanifold NNS distributions show a transition from a Poisson distribution to a Brody distribution at those energy-field parameters.

In parallel magnetic and electric fields $(\beta=0)$ the intramanifold dynamics is regular because of the cylin- 
drical symmetry of the system and the conservation of the $z$-component of the angular momentum. It might be expected that intramanifold chaos increases the more the cylindrical symmetry is broken and becomes strongest in perpendicular crossed fields. The interesting result of this Paper is that this assumption is not true and intramanifold chaos becomes strongest, in our examples, at mutual field orientations around $\beta \approx 60^{\circ}$. Effects of intramanifold chaos are surprisingly weak at $\beta=90^{\circ}$ as can be seen in Figs. 8e and 10e. Probably this cannot be explained by the existence of a discrete symmetry, i.e., the $z$-parity, $\pi_{z}$, in perpendicular crossed fields and further investigations are necessary to understand the mechanism of the increase and decrease of intramanifold chaos.

Implications are that some effects which have been discussed recently for the crossed field atom such as Ericson fluctuations of the continuous photo cross section above threshold [9] and Arnol'd diffusion of the threedimensional classical motion [12] might be much stronger at mutual field orientations around $\beta \approx 60^{\circ}$ than in perpendicular crossed fields. It might be useful to revisit these problems and extend the previous investigations to arbitrary mutual orientations of the external fields.

Up to now experimental investigation on atoms in combined electric and magnetic fields have concentrated on parallel and perpendicular field orientation. If indeed some interesting physical effects are much stronger for special mutual field orientations than in parallel or perpendicular fields it is a challenge to observe these effects experimentally.

\section{ACKNOWLEDGMENTS}

We thank T. Uzer and J. von Milczewski for stimulating discussions. This work was supported by the Deutsche Forschungsgemeinschaft (SFB 237).

[1] H. Friedrich and D. Wintgen, Phys. Rep. 183, 37 (1989).

[2] H. Hasegawa, M. Robnik, and G. Wunner, Prog. Theor. Phys. 98, 198 (1989).

[3] S. Watanabe, in Review of Fundamental Processes and Applications of Atoms and Ions, edited by C. D. Lin (World Scientific, Singapore, 1993).

[4] G. Wiebusch, J. Main, K. Krüger, H. Rottke, A. Holle, and K. H. Welge, Phys. Rev. Lett. 62, 2821 (1989).

[5] G. Raithel, M. Fauth, and H. Walther, Phys. Rev. A 44, 1898 (1991) and Phys. Rev. A 47, 419 (1993).

[6] G. Raithel, H. Held, L. Marmet, and H. Walther, J. Phys. B 27, 2849 (1994).

[7] W. Pauli, Z. Phys. 36, 335 (1926).

[8] E. A. Solov'ev, Sov. Phys. JETP 58, 63 (1983).
[9] J. Main and G. Wunner, Phys. Rev. Lett. 69, 586 (1992) and J. Phys. B 27, 2835 (1994).

[10] M. J. Gourlay, T. Uzer, and D. Farrelly, Phys. Rev. A 47, 3113 (1993); J. von Milczewski and T. Uzer, Phys. Rev. A 56, 220 (1997).

[11] J. von Milczewski, G. H. F. Diercksen, and T. Uzer, Phys. Rev. Lett. 73, 2428 (1994); J. von Milczewski and T. Uzer, Phys. Rev. E 55, 6540 (1997).

[12] J. von Milczewski, G. H. F. Diercksen, and T. Uzer, Phys. Rev. Lett. 76, 2890 (1996).

[13] J. von Milczewski, D. Farrelly, and T. Uzer, Phys. Rev. Lett. 78, 1436 and 2349, and Phys. Rev. A 56, 657 (1997).

[14] V. S. Melezhik, Phys. Rev. A, 48, 4528 (1993).

[15] P. Fassbinder and W. Schweizer, Phys. Rev. A. 53, 2135 (1996).

[16] M. Schwacke, diploma thesis, Ruhr-Universität Bochum (1994).

[17] J. Nutall and H. L. Cohen, Phys. Rev. 188, 1542 (1969).

[18] W. P. Reinhardt, Ann. Rev. Phys. Chem. 33, 223 (1982).

[19] D. Delande, A. Bommier and J. C. Gay, Phys. Rev. Lett. 66, 41 (1991).

[20] T. Ericsson and A. Ruhe, Math. Comput. 35, 1251 (1980).

[21] T. N. Rescigno and V. McKoy, Phys. Rev. A 12, 522 (1975).

[22] P. Epstein, Phys. Rev. 22, 202 (1923).

[23] M. Born, Vorlesungen über Atommechanik (Springer, Berlin, 1925).

[24] P. A. Braun and E. A. Solov'ev, Sov. Phys. JETP 59, 38 (1984).

[25] O. Bohigas and M. J. Giannoni, in Mathematical and Computational Methods in Nuclear Physics, Lecture Notes in Physics, Vol. 209, edited by J. S. Dehesa et al. (Springer, Berlin, 1984).

[26] F. Haake, Quantum Signatures of Chaos, (Springer, New York, 1991).

[27] T. A. Brody, J. Flores, J. B. French, P. A. Mello, A. Pandey, and S. S. M. Wong, Rev. Mod. Phys. 53, 385 (1981). 